\title{
Application of Simulated Annealing in Transit Schedule Synchronization
}

\author{
Vahid Poorjafari, Wen Long Yue, and Nicholas Holyoak
}

\begin{abstract}
Providing temporal coordination among public transport servicesis of vital importance in transit planning, as it has direct impacts on the waiting time imposed on transferring passengers. This task, which is widely recognized as schedule synchronization, is highly complicated by nature since it typically leads to a complex combinatorial optimization problem. This study aims to investigate the capability of simulated annealing algorithm in coping with this problem. A new mathematical programming model is presented for the purpose of minimizing the total transfer waiting time in transit networks. Then, a simulated annealing algorithm is developed and applied to a small-size transit network in order to test the algorithm applicability. The numerical results showed the capability of the algorithm in tackling the transit schedule synchronization problem.
\end{abstract}

Index Terms - Transit, public transport, simulated annealing, schedule.

\section{INTRODUCTION}

In public transport systems, it is almost implausible to connect all origins and destinations with direct lines because of economic reasons. Therefore, transit users often need to take several services to arrive at their destinations. Lack of temporal coordination between arrival and departure of related services at transfer points imposes long waiting times on transferring passengers. Reducing this waiting time, widely known as transfer waiting time, is a crucial step in public transport planning and is the main aim of transit timetable coordination.

Transit schedule coordination, also known as schedule synchronization, concerns with setting the timetables for a transit network by which coordination among services at connecting stops are guaranteed [1]. It is typically accomplished through modifying the existing timetables and shifting the departure/arrival times of transit vehicles for the purpose of minimizing transfer waiting time between related services. As a major stage in transit timetabling, this task is unanimously considered as the most problematic stage for public transport planners [2].

Although the schedulesynchronization is sometimes simplified in practice in the favor of coordination at a few transfer points, network-wide synchronization is a highly difficult problem by nature, as its formulation results in a complex combinatorial optimization problem [3], [4]. In fact, this problem in many cases is a NP-hard problem, which is

Manuscript received July 1, 2014; revised August 26, 2014.

Vahid Poorjafari and Nicholas Holyoak are with the Transport Engineering at the School of Natural and Built Environments, University of South Australia (e-mail: cxqvy002@mymail.unisa.edu.au, Nicholas.holyoak@unisa.edu.au).

Wen Long Yue is with the Barbara Hardy Institute, University of South Australia (e-mail: wen.yue@unisa.edu.au). unlikely to be solved with conventional solution methods [5]. The intractability of this problem relies on the need for searching the optimum solution in a very large search space created by permutation of all possible departure/arrival times of operating transit vehicles in each line. That is, an effective algorithm for finding the exact solution for this problem is very less likely to exist [4]. Hence, the use of approximate methods (e.g. metaheuristic algorithms) which yield near-optimum solutions in a relatively short time could be an alternative in coping with the transit schedule synchronization problem.

This paper aims to investigate the applicability of the simulated annealing, as a powerful metaheuristic, in coping withthis problem. A novel formulation is presented for the purpose of minimizing the total transfer waiting time in transit networks. Restriction of transfer waiting times at all transfer points to a tolerable range is a novelty in the proposed model. Afterwards, the application of the simulated annealingis investigated through a numerical example.

\section{LITERATURE REVIEW}

Coordination of timetables has always been a concern for public transport planners and several approaches have arisen in the literature in order to tackle this complex problem. The most common objective considered in these approaches is minimization of total transfer waiting time imposed on all transferring passengers in transit networks over a scheduling period (e.g. peak/off-peak periods). Although the schedule synchronization problem has been modeled as a quadratic assignment problem in a few cases, the Mixed-Integer Linear Programming (MILP) and Mixed-Integer Nonlinear Programming (MINP) have been two classical forms for this problemin the previous work.

Reference [2] developed a mixed integer programming model for maximizing the number of simultaneous arrivals of buses from different lines at some selected transfer points. Reference [4] improved this model for preventing bus bunching and optimizing passengers' transfer in bus systems. They used Branch and Bound, as well as multi-start iterated local search algorithms to resolve the optimization problem.

Reference [6] also proposed another method for maximizing synchronization between railway lines and facilitating transfers with minimum waiting time. Reference [7] presented a model for synchronizing train lines and feeder buses. The focus is on minimizing transfer time between the services and bus operating costs and developed a penalized objective function to find the optimum sets of frequencies using genetic algorithms. Another approach was proposed in [5] for modifying an existing timetable in order to minimize the total transfer waiting time for the entire of a bus network. Reference [8] developed a mixed integer programming 
model for any type of transit system, as well. They assumed uniform headways and deterministic running times and proposed a method for minimizing the total transfer waiting time using genetic algorithms.

As mentioned above, minimization of total transfer waiting time has been the main aim in most of the previous attempts. This objective may lead to reducing the waiting time at the major transfer points having higher volume of transferring passengers at the cost of imposing long waiting times on the passengers waiting at minor transfer points. In public transportation, however, the satisfaction of all users should be taken into consideration. The proposed model in this study not only aims to minimize the total transfer waiting time, but also restricts all feasible waiting times to a pre-determined endurable range.

\section{MODEl CONSTRUCTION}

Let us consider two transit lines $i$ and $j$ intersecting at transfer point c (Fig. 1-a). Transferring from line $i$ to line $j$ adds transfer time to the passengers' travel time. The transfer time consists of two components, namely, the walking time $\left(w_{i j}\right)$ between the related stops/platforms and the transfer waiting time $\left(t w_{i j}\right)$ for the next vehicle in $j$. The walking time is dependent onthe physical distance between stops/platforms of $i$ and $j$, as well as on the ease of access for different groups of passengers. Nevertheless, the transfer waiting time depends on the temporal coordination between the arrival of transit vehicles in $i$ at $c$ and departure times of vehicles in $j$ from $c$. Fig. 1-b displays the transfer waiting time imposed on different groups of transferring passengers from $i$ to $j$ at $c$.

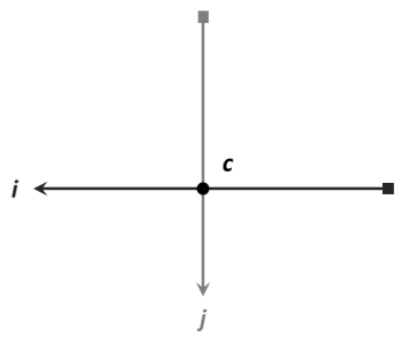

(a). Intersection of two transit lines at a transfer point.

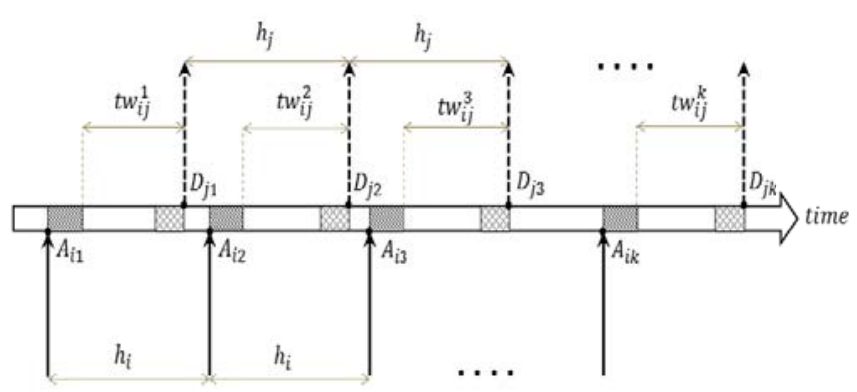

walking time $\longrightarrow$ dwell time $\longrightarrow$ line $(i) \quad-\cdots$ line $(j)$

(b). Transfer waiting time for different groups of passengers.

Fig. 1.Transfer waiting time between two intersecting transit lines.

As shown in this illustration, the first vehicle in $i$ arrives at the transfer point at the time $A_{i l}$. The first vehicle in $j$ departs the transfer point at the time $D_{j l}$, after spending the dwell time $d w_{j}$ at $c$. Therefore, the waiting time imposed on the first group of transferring passengers from $i$ to $j\left(t w_{i j}^{1}\right)$ can be expressed as:

$$
t w_{i j}^{1}=D_{j 1}-A_{i 1}-w_{i j}
$$

Considering the departure time of the second service as the summationof its arrival time at $c\left(A_{j 1}\right)$ and the dwell time, Equation (1) can be rewritten as:

$$
t w_{i j}^{1}=A_{j 1}+d w_{j}-A_{i 1}-w_{i j}
$$

The arrival time of each transit vehicle at the transfer point is a function of the departure time from the first stop $(S)$ and the average running time from the first stop to the transfer point ( $r$ ). Thus, Equation (2) can be expanded as follows:

$$
\left.t w_{i j}^{1}=S_{j}+r_{j}+d w_{j}-S_{i}+r_{i}\right)-w_{i j}
$$

where, $S_{i}$ and $S_{j}$ are the departure times of the first vehicles operating in $i$ and $j$ from the first stops, respectively, and $r_{i}$ and $r_{j}$ denote the average running time from the first stops in $i$ and $j$ to $c$, in order.

The second vehicles in lines $i$ and $j$ arrive at $c$ after the headways $h_{i}$ and $h_{j}$, respectively. Therefore, the transfer waiting time imposed on the second group of transferring passengers from $i$ to $j\left(t w_{i j}^{2}\right)$ is:

$$
\begin{aligned}
& t w_{i j}{ }^{2}=S_{j}+h_{j}+r_{j}+d w_{j}-\left(S_{i}+h_{i}+r_{i}\right)-w_{i j} \\
& =t w_{i j}{ }^{1}+\left(h_{j 1}-h_{i 1}\right)
\end{aligned}
$$

This equation shows that the transfer waiting time incurred by the second groups of transferring passengers is a function of the transfer waiting time for the first group and the difference between the headways of $i$ and $j$. In case the intersecting lines have equal headways, the transfer waiting time is the same for all groups of transferring passengers from $i$ to $j$ over a scheduling period (e.g. peak period). In contrast, $t w_{i j}$ varies over time when the intersecting lines have unequal headways, which is more likely in real-world transit systems.

Investigatingthe variation of transfer waiting time reveals that this variation is cyclic. In other words, the transfer waiting times for different groups of passengers are repeated regularly over a period of time. We callthe period of time covering all feasible transfer waiting times from $i$ to $j$ as transfer cycle $\left(T C_{i j}\right)$ for two intersecting lines $i$ and $j$ and define it as the least common multiple $(\mathrm{lcm})$ of the headways $h_{i}$ and $h_{j}$. Consequently, the number of feasible transfers from $i$ to jover a scheduling period $\left(n_{i j}\right)$ can be determined asTC $C_{i j} / h i$.

Based on Equation (4), the transfer waiting time for the k-th group of transferring passengers from $i$ to $j\left(t w_{i j}^{k}\right)$ can be expressed ast $w_{i j}^{1}+(k-1)\left(h_{j}-h_{i}\right)$. However, the vehicles operating in $j$ are run every $h_{j}$ minutes and the waiting passengers at $\mathrm{c}$ take the earliest vehicle in $j$ arriving at $c$. Hence, the actual transfer waiting time for the $k$-th group of 
transferring passengers can be expressed as follows:

$$
\left.t w_{i j}^{k}=\left[t w_{i j}^{1}+(k-1) \mid h_{j}-h_{i}\right] \bmod h_{j}\right]
$$

The absolute value of $\left(h_{j}-h_{i}\right)$ is used in order to cover the situation in which the second line has a shorter headway than the first line headway. Having Equation (5), it is possible to identify the maximum feasible transfer waiting time imposed on transferring passengers from $i$ to $j$ at $c\left(t w_{i j}^{\max }\right)$ over a scheduling period. Moreover, the total transfer waiting time from $i$ to $j$ at $c\left(T W_{i j}\right)$ can also be calculated using this equation.

As mentioned in the previous section, the main aim of transit timetable synchronization is to minimize the total transfer waiting time incurred by all transferring passengers in a transit network over a scheduling period $(S P)$. Hence, the all transfer waiting times at all transfer points in the transit network should be taken into account and an optimization model should be developed for the purpose of minimizing this value subject to the operational limitations of transit systems. Since the transfer waiting time is a function of departure time from the first stop (Equation (3)), the total transfer waiting time in a transit network can be presented as a function of departure times from the first stops. In fact, transit schedule synchronization aims to find the set of departure times from the first stops by which the total transfer waiting time over $S P$ becomes minimized. In the case of synchronizing existing transit systems, the solution is the set of shifts $(X)$ in the existing departure times from the first stops which lead to minimum total transfer waiting time in a transit network.

In order to develop the mathematical programming model for the timetable synchronization problem, we adopt the following underlying assumptions:

1) Physical characteristics of the transit network, including the lines alignment, stop locationsand average running times between stops are provided.

2) Dwell times, walking times and number of transferring passengers at each transfer point in known.

3) Transferring passengers at each transfer point are uniformly distributed among the feasible transfers between the related lines over $S P$.

4) Headways are known and fixed over $S P$.

From a planning point of view, some transfers are more important than others corresponding to their location, direction and time. For instance, some transfer points play a more important role in transit networks (e.g. intermodal interchanges) and transfers at such points should be favored. Therefore, the importance factor $(I)$ should be considered as a weighting factor in addition to the number of transferring passengers in order to reflect the planning concerns in synchronizing transit timetables.

Considering the transfer waiting time as a function of the shift in the first departure time and the importance factor, the synchronization model for minimizing the total transfer waiting time in a transit network can be proposed as follows:

$$
\min Z=\sum_{i=1}^{N} \sum_{j=1}^{N} \sum_{c=1}^{m} T W_{i j}^{c} \cdot P_{i j}^{c} \cdot I_{i j}^{c} \cdot f_{i j}^{c}
$$

Subject to:

$$
\begin{aligned}
& T W_{i j}^{c}=\sum_{k=1}^{n_{i j}} t w_{i j}^{k c} \\
& \left.t w_{i j}^{k}=\left[t w_{i j}^{1}+(k-1) \mid h_{j}-h_{i}\right] \bmod h_{j}\right] \\
& t w_{i j}^{1 c}=S_{j}+X_{j}+r_{j}^{c}+d_{j}^{c}-\left(S_{i}+X_{i}+r_{i}^{c}\right)-w_{i j}^{c} \\
& t w_{i j}^{c \max }=\max \left\{t w_{i j}^{k c} \mid k \in\left\{1, \ldots . ., n_{i j}\right\}\right\} \\
& P_{i j}^{c}=\frac{h_{i} \times \rho_{i j}^{c}}{60} \\
& t w_{i j}^{1 c} \geq 0 ; \forall i, j \in\{1, \ldots \ldots . . N\} \\
& t w_{i j}^{\operatorname{maxc}} \leq T_{\max } ; \forall i, j \in\{1, N\} \\
& \frac{-h i}{2} \leq X_{i} \leq \frac{h i}{2} \quad ; \forall i \in\{\ldots N\} \\
& X_{i} \in Z ; \forall i \in\{1, \ldots \ldots . . N\}
\end{aligned}
$$

where,

$N:$ number of directional lines in the network

$m:$ number of transfer points in the network

$P_{i j}^{c}$ : number of transferring passenger at each transfer

$f_{i j}^{c}$ : filtering factor for unfeasible transfers (1 if transfer between $\mathrm{I}$ and $\mathrm{j}$ is feasible at $\mathrm{c}$, and 0 otherwise)

$t w_{i j}^{k c}: \mathrm{k}$-th transfer waiting time from $i$ to $j$ at $c$

$t w_{i j}^{1 c}$ : the first transfer waiting time from $i$ to $j$ at $c$

$\rho_{i j}^{c}$ : hourly volume of transferring passengers from $i$ to $j$ at

$c$

$X_{i}$ : shift in the departure time from the first stop in line $i$

In this model, the objective function is the total transfer waiting time in the network considering the importance of transfers. Equations (7), (8), (9) and (11) present the parameters used in the objective function. Constraint (12) guarantees the first transfer from $i$ to $j$ at $c$ to occur andConstraint (13) provides the opportunity to restrict the maximum feasible waiting time to a pre-determined tolerable value $\left(T_{\max }\right)$. Since the shift in the exiting timetable is in minutes, the decision variable $(\mathrm{X})$ can take only integer values (Constraint 15). This parameter is allowed to vary within the range defined in Constraint (14) in order to maintain the existing line frequencies (i.e. no need for adding/removing the existing services).

\section{ApPlication of Simulated AnNealing}

The schedule synchronization model developed in the previous section is a combinatorial optimization problem. The complexity of this model mainly relies on the need to search for the optimum solution in a huge search space made up by combination of all feasible shifts in the departure times from the first stops. It should be noted here that the complexity of this optimization problem increases exponentially by the number of directional lines. Consequently, the problem becomes intractable in the case of 
dealing with huge public transport networks and the application of exact solution methods would be exhaustive. Therefore, employing approximate solution algorithms, which return near-optimum solution in a relatively short computing time, is the only possibility to cope with this problem.

Simulated annealing (SA) is a widely used algorithm to address discrete, as well as continuous optimization problems The key feature of SA is its capability in escaping local optima in hopes of finding a global optimum [9]. This algorithm is a single-solution metaheuristic algorithm which is inspired by an analogy between combinatorial optimization problems and the physical annealing of solids [10]. Its convergence properties, its capability in finding global optimum and its ease of implementation has made SA a popular solution technique over the past years and it is now being used very widely for tackling complex optimization problems arisen in different areas of engineering.

SA starts from a randomly generated initial solution and at each iteration, a trial solution is generated using a neighborhood generating function. If the trial solution is better than the current solution, it is selected as the current solution is replaced by the trial solution. Otherwise, the trial solution is accepted with a probability which typically depends on the difference in objective function value and a control parameter, called temperature. For minimization problems, this probability is defined based on the Metropolis distribution, as follows [10]:

$$
P_{\text {accept }}\left(s, s^{\prime}, T\right)=\left\{\begin{array}{l}
1, \quad \text { if } f\left(s^{\prime}\right)<f(s) \\
\exp \left(\frac{f(s)-f\left(s^{\prime}\right)}{T}\right), \text { otherwise }
\end{array}\right.
$$

where, $P_{\text {accept }}$ is the probability of acceptance, $s$ is the current solution, $s$ ' is the trial solution, $T$ is temperature, and $f(s)$ and $f\left(s^{\prime}\right)$ are the objective function values under the current and the trial solutions, respectively. After sufficient iterations at each temperature, the temperature is lowered and the probability of accepting worse trial solutions decreases so that only better solutions are accepted at low temperatures. If the basic parameters are set properly and the annealing process is relatively slow, SA is promising to yield the global optimum.

In this study, a simulated annealing algorithm was developed using Matlab programming language in order to solve the schedule synchronization model proposed in the previous section. Each solution is structured as a string of integers representing the shifts in the departure times from the first stops. The best solution, therefore, is the string by which the total transfer waiting time in the network becomes minimized. In this algorithm, Constraints (14) and (15) are satisfied directly through the neighborhood generation function. Constraints (12) and (13) are also dealt through the penalty method. A huge penalty is applied to unfeasible solutions generated by the annealing function in order to reduce their chance to be selected as the final solution. The amount of penalty is selected relative to the amount of violation, as shown in the following penalty function:

where, $Z_{p}$ is the penalized value of the objective function, and $P_{1}$ and $P_{2}$ are the penalty factors. It should be noted that the values of penalty factors are case-dependent and should be selected properly in order to avoid unfeasible solutions.

$$
\begin{aligned}
Z_{p}= & Z+\sum_{i=1}^{N} \sum_{j=1}^{N} \sum_{c=1}^{m} P_{1}\left(t w_{i j}^{1 c}\right)^{2} \\
& +\sum_{i=1}^{N} \sum_{j=1}^{N} \sum_{c=1}^{m} P_{2}\left(t w_{i j}^{c \max }-T_{\max }\right)^{2}
\end{aligned}
$$

\section{A NumERICAL EXAMPLE}

This section aims to examine the capability of the proposed synchronization model and the simulated annealing algorithm in reducing transfer waiting time in transit networks through an example. The example is a small-size transit network comprising a bidirectional trunk line intersecting two bidirectional feeder lines at two transfer points $\mathrm{A}$ and $\mathrm{B}$ (Fig. 2). The headways and the average running time on each network segment are given. Table I also presents the transfer matrix, which indicates the number of transferring passengers among the lines at the transfer points. It is assumed that under the existing timetable, all the transit vehicles depart the first stops at 8:00 am (Table II). In this example, the focus is on the waiting time imposed on the passengers transferring from the trunk line to the feeders and vice versa and it is intended to modify the existing timetable (i.e. departure times from the first stops) for the purpose of minimizing the total transfer waiting time in the network over one peak hour (8:00-9:00 am).

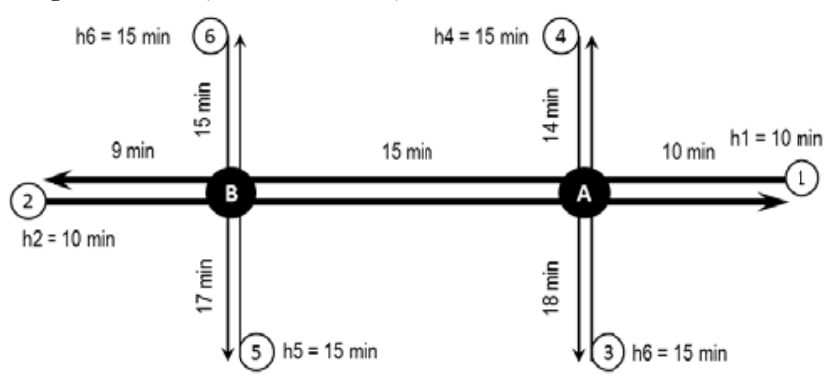

Fig. 2. Intended transit network.

TABLE I: HOURLY VOLUME OF TRANSFERRING PASSENGERS

\begin{tabular}{||c|c|c|c|c|c|c||}
\hline \hline & 1 & 2 & 3 & 4 & 5 & 6 \\
\hline 1 & - & - & 45 & 50 & 30 & 35 \\
\hline 2 & - & - & 30 & 40 & 60 & 55 \\
\hline 3 & 60 & 20 & - & - & - & - \\
\hline 4 & 50 & 35 & - & - & - & - \\
\hline 5 & 35 & 60 & - & - & - & - \\
\hline 6 & 30 & 40 & - & - & - & - \\
\hline
\end{tabular}

TABLE II: DEPARTURE TIMES FROM THE FIRST STOPS UNDER THE CURRENT SCHEDULE

\begin{tabular}{||c|c|c|c|c|c||}
\hline 1 & 2 & 3 & 4 & 5 & 6 \\
\hline $8: 00$ & $8: 00$ & $8: 00$ & $8: 00$ & $8: 00$ & $8: 00$ \\
$8: 10$ & $8: 10$ & $8: 15$ & $8: 15$ & $8: 15$ & $8: 15$ \\
$8: 20$ & $8: 20$ & $8: 30$ & $8: 30$ & $8: 30$ & $8: 30$ \\
$8: 30$ & $8: 30$ & $8: 45$ & $8: 45$ & $8: 45$ & $8: 45$ \\
$8: 40$ & $8: 40$ & & & & \\
$8: 50$ & $8: 50$ & & & & \\
\hline
\end{tabular}

Additional assumptions in this example are as follows:

1) Dwell time at the transfer points $=1$ minute

2) Walking time at each transfer $=1$ minute

3) Maximum allowable transfer waiting time $\left(T_{\max }\right)=12$ 
minutes

4) All the transfers are considered at the same level of importance (i.e. $I_{i j}^{c}=1$ ).

Since the performance of simulated annealing algorithm is highly affected by its basic parameters and operators, a range of sensitivity analysis was conducted on the algorithm in this study. Based on this analysis, the penalty factors are selected as $\mathrm{P}_{1}=\mathrm{P}_{2}=1$. The initial temperature was selected 1000 andthe temperature schedule function was also chosen as follow:

$$
T=T_{0} 0.95^{k}
$$

where, $T$ is the current temperature, $T_{0}$ is the initial temperature and $k$ is the annealing factor. The annealing function, which generates the trial solutions, was also developed so that only integers were assigned to the decision variables.

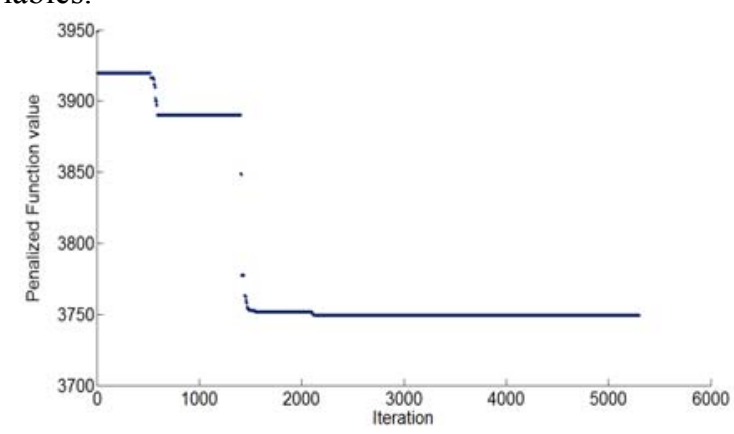

Fig. 3. Improvement in objective function value.

Using a $1.83 \mathrm{GHz} \mathrm{CPU}$ and $4 \mathrm{~GB}$ of RAM, the synchronization model was solved for the intended network after 5303 iterations in $104 \mathrm{sec}$. Fig. 3 displays the value of the penalized objective function versus iterations. As can be seen in this graph, the improvement in the objective function occurred faster at the higher temperatures and it became slower at the lower temperatures. The best solution found by the algorithm is as follows:

$$
X^{*}=[-5,1,-3,-2,6,-5]
$$

According to this solution, the departure times from the first stops were altered and the modified timetable was created (Table III). Fig. 4 demonstrates the reduction in transfer waiting time under the synchronized schedule over one peak hour.

TABLE III: DEPARTURE TIMES FROM THE FIRST STOPS UNDER THE MODIFIED SCHEDULE

\begin{tabular}{||c|c|c|c|c|c||}
\hline 1 & 2 & 3 & 4 & 5 & 6 \\
\hline $7: 55$ & $8: 01$ & $7: 57$ & $7: 58$ & $8: 06$ & $7: 55$ \\
$8: 05$ & $8: 11$ & $8: 12$ & $8: 13$ & $8: 21$ & $8: 10$ \\
$8: 15$ & $8: 21$ & $8: 27$ & $8: 28$ & $8: 36$ & $8: 25$ \\
$8: 25$ & $8: 31$ & $8: 42$ & $8: 43$ & $8: 51$ & $8: 40$ \\
$8: 35$ & $8: 41$ & & & & \\
$8: 45$ & $8: 51$ & & & & \\
\hline
\end{tabular}

As shown in Fig. 4-a, transfer waiting time at the transfer points $\mathrm{A}$ and $\mathrm{B}$ decreased by 14 and 18.7 percent, respectively, and the total transfer waiting time in the network declinedby $16.4 \%$, from 3933 minutes to 3288 minutes under the modified timetable. In other words, the modification of the existing timetable resulted in saving 645 minutes just over one peak hour. Fig. 4-b also shows that the waiting time incurred by each transferring passengers in the network decreased by 5.83 minutes to 4.87 minutes, on average. In fact, the modification of the existing timetable led to reducing the average waiting time by almost 1 minute for each passenger.

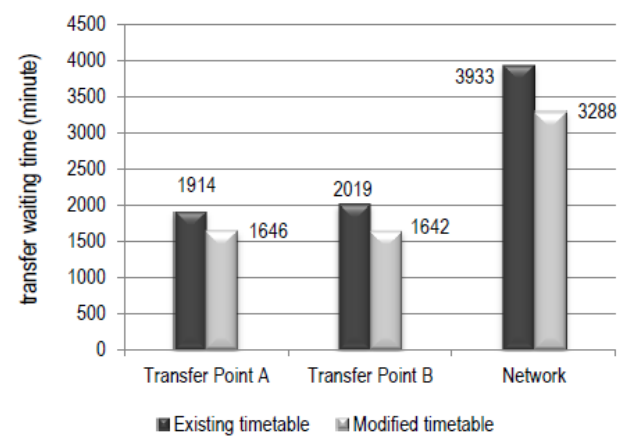

(a). Total transfer waiting time.

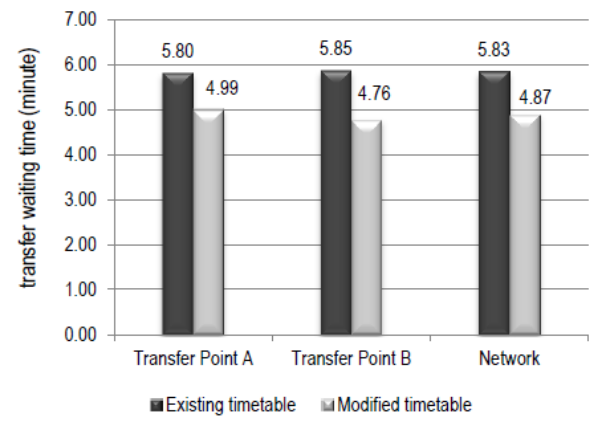

(b). Average transfer waiting time for each transferring passenger.

Fig. 4. Impacts of timetable synchronization on transfer waiting time.

In addition to the reduction in the total transfer waiting time, synchronization of the existing timetable reduced long waiting times at the transfer points. Under the existing timetable, $10.2 \%$ of the waiting times exceed $\mathrm{T}_{\max }(12$ minutes). However, this percentage declined to $6.1 \%$ under the modified timetable due to restricting transfer waiting times to a pre-determined endurable waiting time (Constraint 13). In fact, timetable synchronization not only reduced the total transfer waiting time in the network, but also prevents imposing long and unendurable waiting times on the waiting passengers. Overall, the proposed schedule synchronization method resulted in substantial improvements in the system performance and the quality of transfers in the intended network.

\section{CONCLUSION}

This paper presented the application of a simulated annealing algorithm in coping with the transit schedule synchronization problem. A new optimization model was proposed for the purpose of minimizing the waiting time imposed on the transferring passengers in public transport systems. Restricting the transfer waiting time to a tolerable range is a novelty in this formulation. A simulated annealing algorithm was also developed in order to solve the proposed optimization problem. Application of the method to a transit network revealed that simulated annealing is a capable solution algorithm for tackling the transit schedule synchronization problem, which is a complex optimization problem by nature.

Since the performance of simulated annealing is highly dependent on its basic parameters and operators, further 
sensitivity analysis on these features could improve the efficiency of this solution technique in terms of dealing with the timetable synchronization problem. Applying other metaheuristics and comparing the results to the developed simulated annealing algorithm could also be an extension for this work.

\section{REFERENCES}

[1] L. Castelli, R. Pesenti, and W. Ukovich, "Scheduling multimodal transportation systems," European Journal of Operational Research, vol. 155 , no. 3, pp. 603-615, 2004.

[2] A. Ceder, B. Golany, and O. Tal, "Creating bus timetables with maximal synchronization," Transportation Research Part A: Policy and Practice, vol. 35, no. 10, pp. 913-928, 2001.

[3] V. Guihaire and J. K. Hao, "Transit network design and scheduling: a global review," Transportation Research Part A: Policy and Practice, vol. 42, no. 10, pp. 1251-1273, 2008 .

[4] O. J. I. Rojas and Y. A. R. Solis, "Synchronization of bus timetabling," Transportation Research Part B: Methodological, vol. 46, no. 5, pp. 599-614, 2012.

[5] F. Cevallos and F. Zhao, "Minimizing transfer times in a public transit network with genetic algorithm," Transportation Research Record: Journal of the Transportation Research Board, vol. 1971, no. 1, pp. 74-79, 2006.

[6] R. C. W. Wong and J. M. Y. Leung, "Timetable synchronization for mass transit railway," presented at the 9th International Conference on Computer-Aided Scheduling of Public Transport (CASPT), San Diego, CA, August 9-11, 2004.

[7] P. Shrivastava and S. L. Dhingra, "Development of coordinated schedules using genetic algorithms," Journal of Transportation Engineering, vol. 128, no. 1, pp. 89-96, 2002.

[8] Y. Shafahi and A. Khani, "A practical model for transfer optimization in a transit network: model formulations and solutions," Transportation Research Part A: Policy and Practice, vol. 44, no. 6, pp. $377-389,2010$
[9] F. Glover and G. A. Kochenberger, Handbook of Metaheuristics, US Kluwer Academic Publishers, 2003, ch. 10, pp. 287-291.

[10] M. Dorigo and T. Stutzle, Ant Colony Optimization, US: The Massachusetts Institute of Technology, 2004, ch. 2, pp. 47-49.

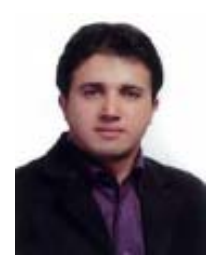

Vahid Poorjafari is a PhD candidate of Transport Engineering at the University of South Australia. His background includes transit planning, urban transportation, transport infrastructure design, system analysis and optimization in transportation systems. $\mathrm{He}$ has published several papers in refereed journals and conference proceedings and currently, he is conducting research on transit systems operation.

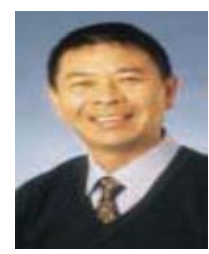

Wen Long Yue is a senior lecturer and the program director of Transport Engineering at the University of South Australia. His professional activities, industry consultancy activities, teaching, academic research and research student supervision interests span in all the aspects of transport systems engineering. $\mathrm{He}$ is an expert in simulation modeling for transport systems and parking systems. Dr. Wen Long Yue lectures in transport planning and traffic engineering at postgraduate and undergraduate levels. His research interests include the safety and operational characteristics of signalized intersections, the analysis procedures of accidents, the operation of public transport systems, transport survey design, logistics operation and management.

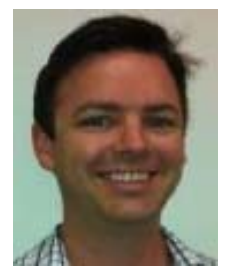

Nicholas Holyoak is a research fellow with the University of South Australia specializing in the Transport Systems field. His research interests include travel behavior, modeling travel demand, network analysis, survey design, deployment and data analysis, sustainable transport and electric vehicles. $\mathrm{He}$ currently lectures in the fields of road design and traffic management. 